\title{
Topographic Mapping of Retinal Function with the SLO-mfERG under Simultaneous Control of Fixation in Best's Disease
}

\author{
Gunther Rudolph Petros Kalpadakis \\ Eye Clinic, Ludwig-Maximilians-University, Munich, Germany
}

\section{Key Words}

Retina - Electroretinography - Best's disease - Fixation . SLO-mfERG · Scanning laser ophthalmoscope

\begin{abstract}
Purpose: To introduce the scanning laser ophthalmoscope-evoked mfERG (SLO-mfERG) as a new method to measure focal retinal function. Methods: Sixty-two healthy individuals and 12 patients with Best's disease were examined. mfERGs were recorded using a scanning laser ophthalmoscope as a stimulator and trigger device (He-Neon $632.8 \mathrm{~nm}$ ) as well as a fundus-monitoring system (Infrared $730 \mathrm{~nm}$ ). Results: Amplitudes in the central concentric area were found to be significantly lower in patients with Best's disease than in healthy controls, while no significant differences were found for the more peripheral areas. Conclusion: SLO-mfERG is a reliable new technique for topographic mapping of retinal function under simultaneous control of fixation.
\end{abstract}

Copyright $\odot$ 2003S. Karger AG, Basel

\section{Introduction}

Electroretinography proceeded from the Ganzfeld ERG [1-3] and EOG [4, 5] which are precise diagnostic tools in generalized retinal diseases, to the multifocal ERG (mfERG) [6-11], which allows mapping of focal retinal function. With the implementation of the visual evoked response imaging system using the m-sequence stimulation technique, a possibility for topographic mapping of retinal function was created. In this study we used a scanning laser ophthalmoscope to compare the SLOevoked multifocal ERGs (SLO-mfERG) from patients with Best's disease with those of healthy individuals (fig. 1). The purpose of this study was to assess the feasibility of this method in registering focal retinal dysfunction under simultaneous control of fixation. The latter is especially necessary in patients with eccentric fixation, e.g. in patients with scars on the posterior pole in the vitelliruptive stage, in order to correlate amplitudes and measured retinal areas reliably.

\section{Methods}

The scanning laser ophthalmoscope (Rodenstock, Munich, Germany) was used as a stimulator and trigger unit and as a fundusmonitoring unit. To record an mfERG, a confocal scanning laser ophthalmoscope was connected to a mfERG device (RETIscan,

\begin{tabular}{ll}
\hline KARGER & $\begin{array}{l}\text { ( ) 2003 S. Karger AG, Basel } \\
\text { 0030-3755/03/2172-0154\$19.50/0 }\end{array}$ \\
$\begin{array}{l}\text { Fax +4161306 12 34 } \\
\begin{array}{l}\text { E-Mail karger@karger.ch } \\
\text { www.karger.com }\end{array}\end{array}$ & $\begin{array}{l}\text { Accessible online at: } \\
\text { www.karger.com/oph }\end{array}$
\end{tabular}

Gunther Rudolph, MD

Eye Clinic, Ludwig-Maximilians-University

Mathildenstrasse 8, D-80336 München (Germany)

Tel. +49 8951603800, Fax +49 8951604569

E-Mail Guenther.Rudolph@ak-i.med.uni-muenchen.de 


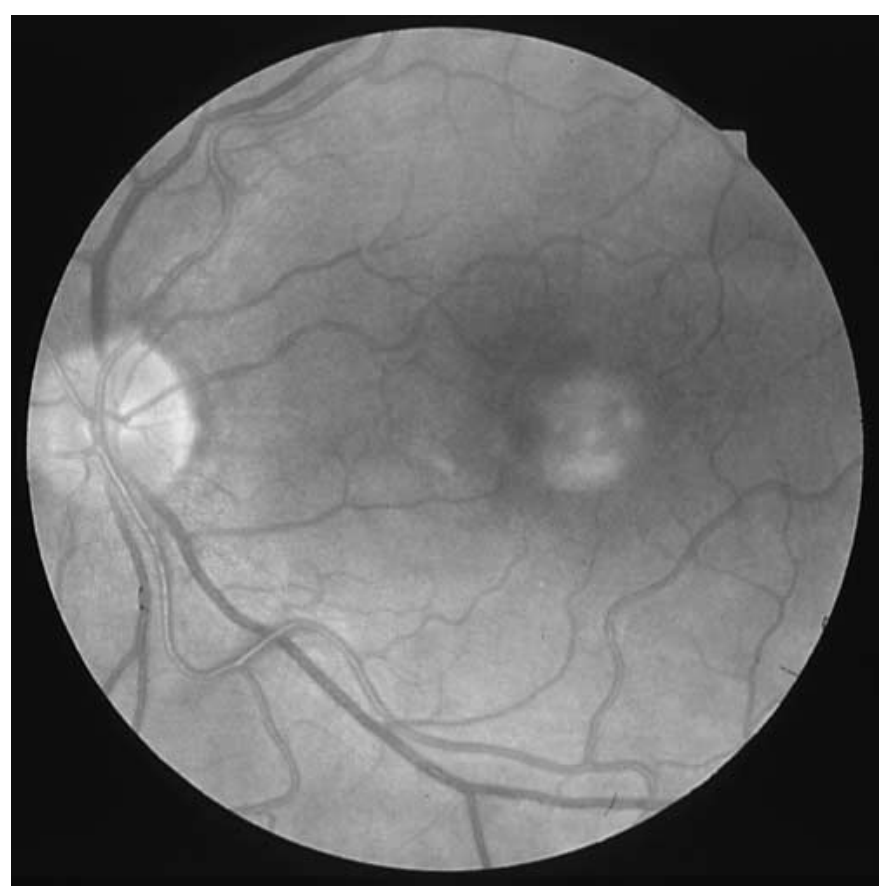

Fig. 1. Fundus photography of a patient with Best's disease in the vitelliruptive stage.

Roland Consult, Wiesbaden, Germany). The visual stimulus, consisting of a matrix of hexagonal areas flickering simultaneously according to a short $\mathrm{m}$-sequence, is projected directly onto the retina, covering a visual angle of $12^{\circ}$ or a diameter of $24^{\circ}$, respectively (fig. 2a, b) [12-14]. We used a matrix of 61 hexagonal elements, generated by a helium-neon laser $(632.8 \mathrm{~nm})$ modulated via an acoustooptic modulator. The helium-neon laser fulfills the criteria for safety according to DIN VDE 0837/IEC 825. The signals were bandpass filtered $(15-100 \mathrm{~Hz})$ and amplified by a 2-channel amplifier with a common mode reaction of $>110 \mathrm{~dB}$ and a sensitivity of $1-20 \mu \mathrm{V} / \mathrm{div}$. The signal of each hexagonal field was calculated by cross-correlation analysis between the m-sequence and the response. The matrix was presented with all hexagonal areas having the same size, i.e. without any scaling of the stimulus matrix. The 61 elements change from red to black or vice versa according to a complete cycle of a binary msequence. Multi-input stimulation technique is a prerequisite to stimulate all areas with equal luminance at the same temporal sequence. The stimulus pattern was generated by a helium-neon laser and the study participants were asked to gaze at a centrally positioned cross. In case of eccentric fixation, the fixation cross can be shifted to another position than the central hexagonal element in order to center the stimulus matrix on the posterior pole of the eye. Simultaneous funduscopy and fixation control was possible by using an infrared diode laser $(730 \mathrm{~nm})$.

\section{Patients and Controls}

In this study, 12 patients with Best's disease were compared with 62 healthy controls by SLO-mfERG. Seventy-four subjects were examined altogether (age range 26-50 years; median 34 years).
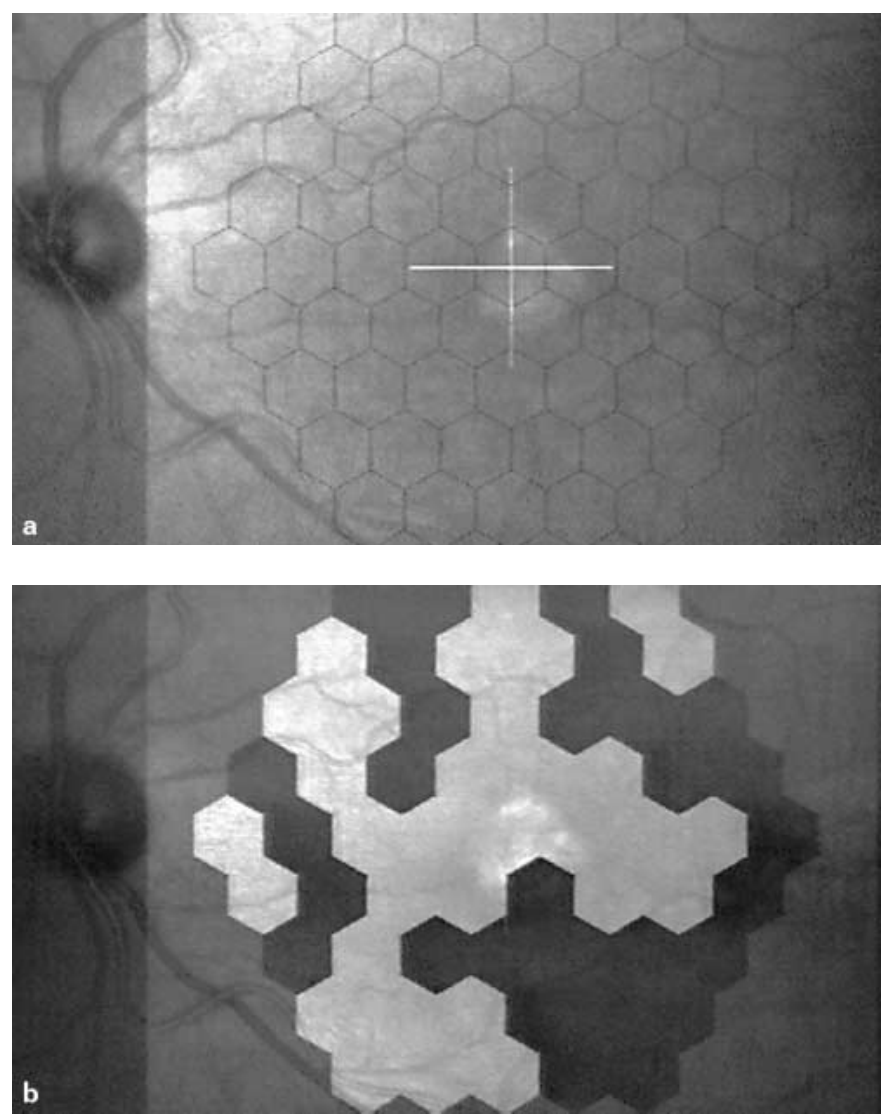

Fig. 2. a Fundus and the overlaying matrix with 61 hexagonal elements. All hexagonal elements are of equal size. $\mathbf{b}$ The stimulation matrix shows a nonrandom pattern at a certain moment.

The criteria for the nonaffected participants included in the study were an age of over 18, visual acuity of 1.0 with or without correction, and contact lens tolerance. Exclusion criteria for both groups were media opacities, a history of ocular trauma, diabetic retinopathy, age-related macular degeneration or glaucoma. Ophthalmic examination included slit lamp examination, measurement of visual acuity, funduscopy and fundus photography. The pupils were not dilated. Refractive abnormalities were corrected. Jet contact lens electrodes (Universa S.A., La Chaux-de-Fonds, Switzerland) were used and the reference electrode was placed directly at the lateral canthus. This research adhered to the tenets of the Declaration of Helsinki and informed consent was obtained from all participants.

\section{Results}

In order to quantify the electrophysiological results, the responses were grouped by retinal eccentricity in 5 ring areas. To summarize the results, boxplots showing the 25 and $75 \%$ percentile of amplitudes were calculated 


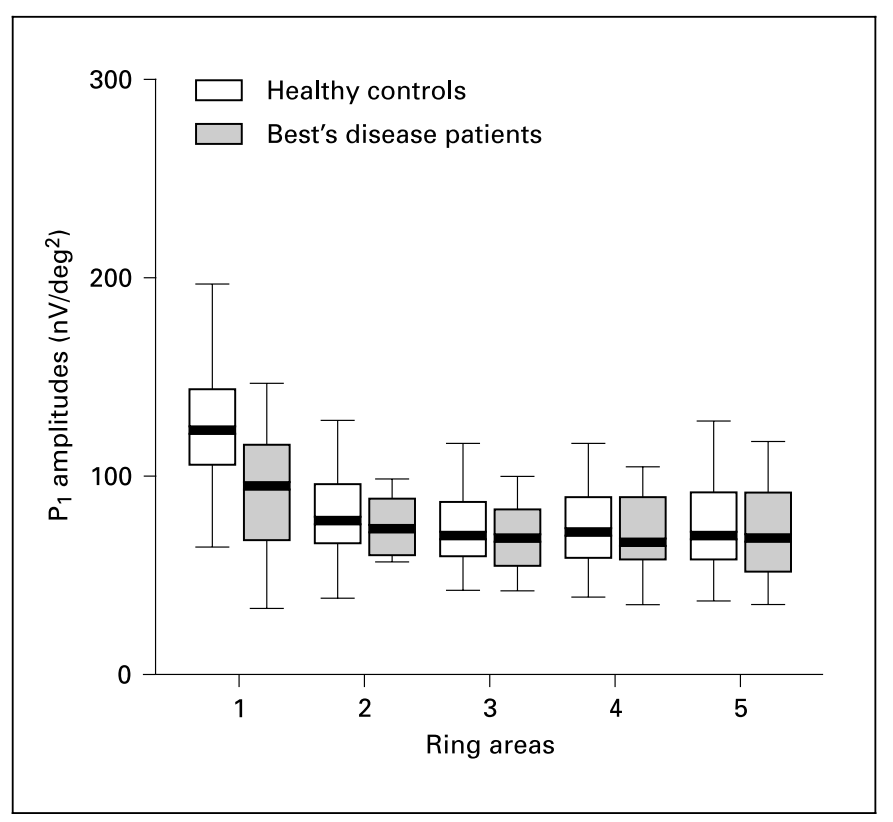

Fig. 3. Boxplots of $P_{1}$ amplitudes in Best's patients $(n=12)$ and healthy controls $(n=62)$.

Table 1. Statistical values of the $P_{1}$ amplitudes $\left(\mathrm{nV} / \mathrm{deg}^{2}\right)$

\begin{tabular}{lrrrrr}
\hline & Ring 1 & Ring 2 & Ring 3 & Ring 4 & Ring 5 \\
\hline Controls & & & & & \\
Mean & 126.9 & 80.8 & 73.3 & 75.3 & 75.5 \\
Median & 123.6 & 77.6 & 70.4 & 72.9 & 70.7 \\
SD & 31.1 & 19.6 & 16.3 & 19.2 & 22.1 \\
CV & 24.5 & 24.2 & 22.2 & 25.4 & 29.2 \\
\hline Best's disease & & & & & \\
Mean & 91.8 & 75.0 & 70.6 & 71.6 & 72.9 \\
Median & 95.8 & 73.9 & 69.7 & 67.6 & 69.6 \\
SD & 31.5 & 15.0 & 18.4 & 21.9 & 25.3 \\
CV & 34.3 & 20.0 & 26.0 & 30.5 & 34.7 \\
\hline
\end{tabular}

$\mathrm{CV}=$ Coefficient of variation

for both groups (patients with Best's disease and healthy controls; fig. 3). The mean and median values as well as the standard deviation, the interquartile range and the coefficient of variation of the central hexagonal element and the rings were calculated for each group. Our data were tested for and were found inconsistent with the normality assumption (using the Kolmogorov-Smirnov test). the significance level was set at $\mathrm{p}=0.05$ and the MannWhitney test was applied.
Statistical differences between patients with Best's disease and controls were found for the amplitudes of the central hexagonal element or ring 1, respectively (table 1). They were significantly higher in controls (ring 1: MannWhitney U: $159.000(\mathrm{p}<0.05), \mathrm{M}$ (normals) $=126.9, \mathrm{SD}=$ 31.1; $\mathrm{M}($ Best $)=91.8, \mathrm{SD}=31.5)$. The amplitudes and latencies of ring areas $2-5$ showed no significant differences (fig. 4a, b).

The coefficient of variation (or percentage error) reflects the relative scatter of data in respect to the mean and therefore the imprecision in our estimates. It is calculated as $(\mathrm{SD} /$ mean value of set $) \times 100$. In our data set, the coefficient of variation of the response density of the central hexagonal element is higher in patients with Best's disease than in healthy controls (table 1).

\section{Discussion}

The SLO-mfERG is a new technical approach to evaluate retinal function by projecting a helium-neon laser stimulus directly onto the retina. Moreover, the presence of an infrared diode laser allows simultaneous funduscopy, control of fixation and imaging of the hexagonal matrix during the examination. This is important for all patients in whom fixation control is difficult, e.g. in patients with macular dystrophies. In case of unstable or eccentric fixation, the matrix would not really cover predefined retinal areas and thus produce results that would not represent the underlying retinal pathology. The central response maximum would be shifted to more peripheral areas while the amplitudes in the center are going to appear decreased.

The importance of appropriate fixation for a reliable stimulation-response correlation analysis has been lately shown using mfERG recordings and SLO-microperimetry [15-16]. Moreover, in a recent study where mfPERG recordings were obtained with an eye tracker from normal individuals, it emerged that $44 \%$ fixated inadequately [17]. Loss of fixation would lead to a wrong correlation of the recorded amplitudes to the expected measured retinal areas. The SLO allows continuous and invariably successful visualization of fixation. The patient can be encouraged and recordings during poor fixation can be discarded. Furthermore, in case of eccentric fixation, the fixation cross can be so moved that, under direct fundus control, the stimulus pattern can be centered on the posterior pole of the eye.

mfERG is a technique capable of measuring retinal function of the posterior pole with high spatial resolution. 
Fig. 4. a Plots from the 61 hexagonal elements of $\mathrm{P}_{1}$ amplitudes of a patient with Best's disease. $\mathbf{b}$ Concentric ring grouping of $\mathrm{P}_{1}$ amplitudes of a patient with Best's disease. Amp. $=$ Amplitude; Lat. = latency. a Amplitudes b
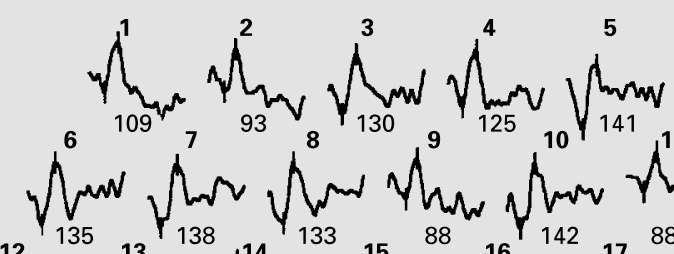

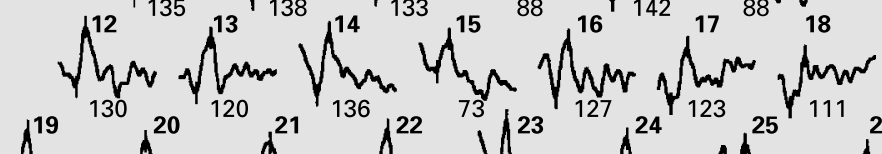
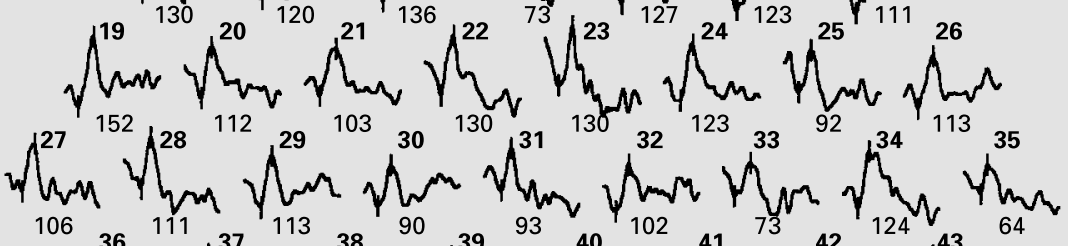

106

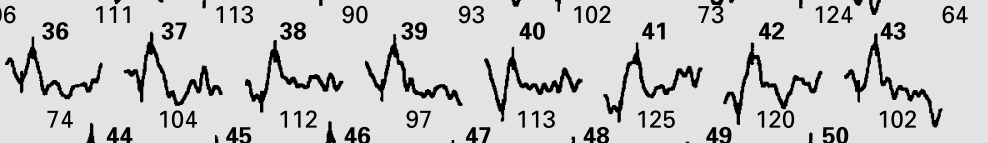

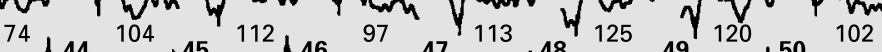

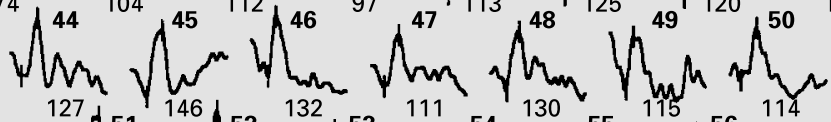

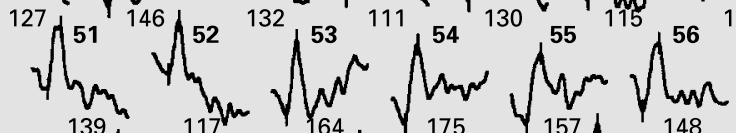

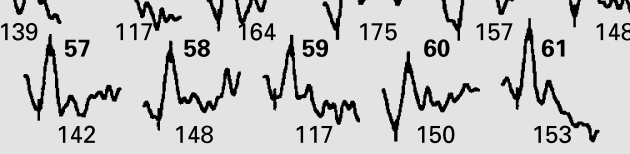
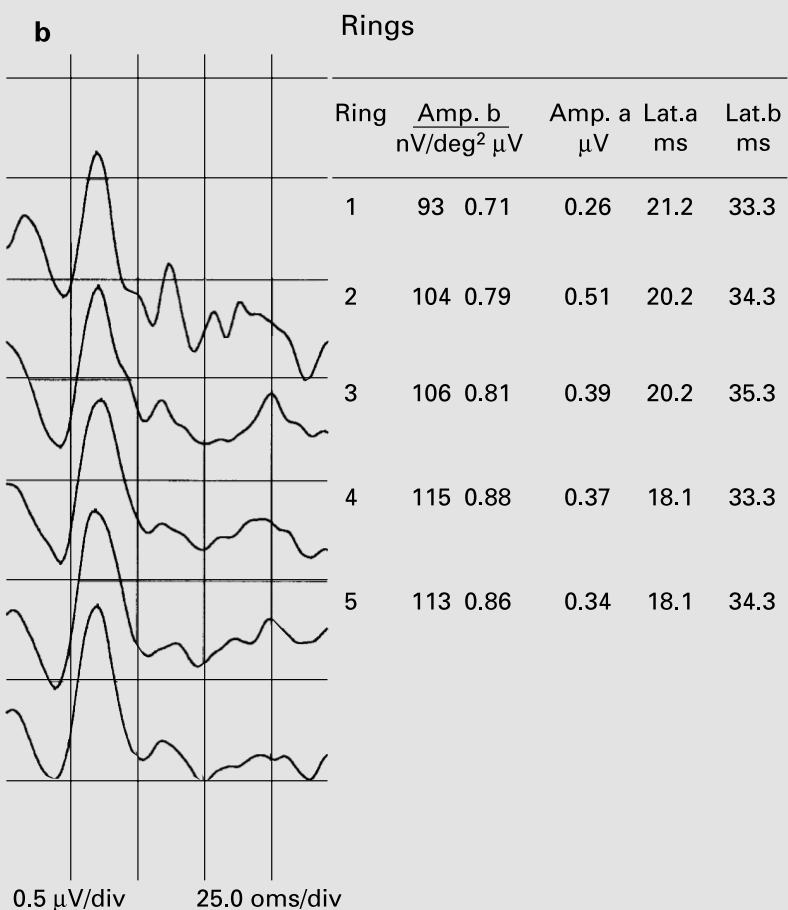
By multifocal stimulation and the simultaneous measurement of all the focal responses, a strict correlation of any given signal to a corresponding field exists. Although the main advantage of the mfERG is its spatial resolution, concentric ring grouping around the innermost hexagon of the stimulation array is a feasible way to obtain reliable results. In concentric retinal diseases, an averaging of concentric rings allows for a better comparison between responses obtained from the central retina and those derived from more eccentric parts [17]. By using short msequences, the quality of each measurement cycle can be evaluated separately, and any cycle in which significant artefacts occur can be discarded [14]. However, this may not be enough to obtain reliable results in cases where loss of foveal fixation exists. In three patients an eccentric presentation of the fixation cross was necessary to center the stimulus matrix to the posterior pole of the eye. The possibility to center the stimulus matrix and to control fixation is a prerequisite to obtain reliable results, especially in patients with macular dystrophies.

Best's vitelliform macular dystrophy is an autosomal dominant macular dystrophy with lipofuscin-like deposits at the posterior pole of the eye. Genetically, the locus for Best's disease has been mapped to chromosome 11q13 [18]. However, the expression mode of this gene is not yet uncovered. The phenotype varies considerably and is characterized by the pre-vitelliform stage with RPE defects, the egg-yolk stage with yellow material in a round smooth cyst, the scrambled egg stage, the pseudohypopyon appearance, and finally the atrophic stage complicated by scars and sometimes subretinal neovascularization [19-22]. Multifocal vitelliform lesions are rare but not uncommon [23]. From the histopathological point of view Best's disease seems to be a generalized retinal pigment epithelial disorder with accumulation of lipofuscin-like material within the RPE, within the macrophages in the subretinal space and within the choroid $[24,25]$. These features can also be demonstrated in the peripheral retina. These observations are useful to explain findings in electrophysiology: the reduced Arden ratio of EOG examination in Best's disease is one of the most important results in the diagnosis of the disease [26]. The EOG is also helpful to distinguish Best's disease from pseudovitelliform macular dystrophy [27]. As a dystrophy of the retinal pigment epithelium results in an inability to process the metabolic products of the neuroreceptors, retinal dysfunction should be more profound where the size of these products is of bigger extent, especially in the macula region.

As known from the conventional mfERG, response density decreases markedly from the foveal region to- wards the periphery. This distribution of response density corresponds approximately to the anatomical occurrence of cone distribution of the posterior pole [12, 13, 28, 29]. In patients with Best's disease, a significant reduction of response density could be demonstrated in the center of the stimulus array. Retinal dysfunction leads to profound amplitude reduction of the central hexagonal element in the SLO-mfERG results of these patients. As expected, the strength of the recorded amplitudes of the more peripheral areas does not differ significantly between Best's disease patients and normal individuals [30].

The high coefficient of variation of central hexagonal element amplitudes in patients with Best's disease (table 1) can be explained through the loss of fixation in these patients. However, it raises questions about the measurements' reliability. Fixation loss (through foveal displacement) shifts the central hexagonal element to a more peripheral retinal area, and for a short but variable time period destroys the correlation between recorded amplitude and field location. Part of the response attributed to the inner ring will also be generated by the fovea, which would otherwise be covered by the central hexagonal element. Our measurements were made under simultaneous fundus visualization and direct fixation control, and still the coefficient of variation implies that such results contain significant errors. Fixation variations can cause problems in patients as well as in healthy individuals. When performing the examination with the monitor version, these fixation control options are not available. Eyetracking, among other efforts to solve this problem, cannot be a reliable solution because absence of eye movement does not absolutely mean proper fixation of the eye to the presented target (fixation cross). Thus, when the above-mentioned precautions are not available, it is important to remember that scaling may introduce significant difficulties in obtaining reliable data $[15,31]$.

While fixation loss cannot be prevented, especially in patients with loss of central retinal function, the value of our fixation monitoring system depends on the possibility of continuous visualization of fixation during the examination. Fixation depending on concentration and motivation can, on the one hand, be positively affected and on the other hand objectively evaluated by using the SLO direct fundus control method in order to record mfERGs.

In conclusion, it seems that fundus monitoring can help overcome the problem of fixation instability sufficiently. SLO-mfERG is a new promising technique to investigate retinal and, in particular, macular function under fixation control. 


\section{References}

1 Carr RE, Siegel IM: In: Electrodiagnostic Testing of the Visual System: A Clinical Guide. Philadelphia, Davies, 1990, pp 3-47.

2 Heckenlively JR, Weleber RG, Arden GB Testing levels of the visual system; in Heckenlively JR, Arden GB (eds): Principles and Practice of Clinical Electrophysiology of Vision. St. Louis, Mosby Yearbook, 1991, pp 485-493.

3 Marmor M, Zrenner E: Standard for clinical electroretinography (1994 update). Doc Ophthalmol 1995;89:199-210.

4 Arden GB, Carter RM, MacFarlan A: Pattern and Ganzfeld electroretinograms in macular disease. Br J Ophthalmol 1984;68:878-884.

5 Arden GB, Vaegan, Hogg CR: Clinical and experimental evidence that the pattern electroretinogram (PERG) is generated in more proximal retinal layers than the focal electroretinogram (FERG). Ann NY Acad Sci 1982;388: 580-607.

6 Hood DC, Seiple W, Holopigian K, Greenstain $\mathrm{V}$ : A comparison of the components of the multifocal and full-field ERGs. Vis Neurosci 1997; 14:533-544.

7 Kretschmann U, Schlote T, Stübiger N, Gendo K, Hipp E, Zrenner E: Multifokale Elektroretinographie bei erworbenen Makularfunktionsstörungen. Klin Monatsbl Augenheilkd 1998; 212:93-100

8 Bock M, Andrassi M, Belitsky L, Lorenz B: A comparison of two multifocal ERG systems. Doc Ophthalmol 1999;97:157-178.

9 Keating D, Parks S, Evans A: Technical aspects of multifocal ERG recording. Doc Ophthalmol 2000;100:77-98.

10 Kretschmann U, Bock M, Gockeln R, Zrenner E: Clinical applications of multifocal electroretinography. Doc Ophthalmol 2000;100:99113.
11 Seeliger MW, Narfström K, Reinhard J, Zrenner E, Sutter E: Continuous monitoring of the stimulated area in multifocal ERG. Doc Ophthalmol 2000;100:167-184.

12 Curcio CA, Sloan Jr. KR, Kalina RE, et al: Human photoreceptor topography. J Comp Neurol 1990;292:497-523.

13 Curcio CA, Sloan Jr. KR, Packer O, et al: Distribution of cones in human and monkey retina. Individual variability and radial asymmetry. Science 1987;236:579-582.

14 Sutter EE: A deterministic approach to nonlinear system analysis; in Printer RB, Nabet B (eds): Nonlinear Vision. Cleveland, CRC Press, 1992, pp 171-220.

15 Rohrschneider K, Bueltmann S: Correlation between fundus perimetry and multifocal ERG using the scanning laser ophthalmoscope (abstract). Invest Ophthalmol Vis Sci 2001;42: S69.

16 Chisholm JA, Keating D, Parks S, Evans AL: The impact of fixation on the multifocal electroretinogram. Doc Ophthalmol 2001;102: 131-139.

17 Parks S, Keating D, Williamson $\mathrm{TH}$, et al: Functional imaging of the retina using the multifocal electroretinograph: A control study. Br J Ophthalmol 1996;80:831-834.

18 Stone EM, Nichols BE, Streb LM, et al: Genetic linkage of vitelliform macular degeneration (Best's disease) to chromosome 11q13. Nature Genet 1992;1:246-250.

19 Krill AE, Morse PA, Potts AM, Klein BA: Hereditary vitelliruptive macular degeneration. Am J Ophthalmol 1966;61:1405-1415.

20 Miller SA, Bresnick OH, Chandra SR: Choroidal neovascular membrane in Best's vitelliform macular dystrophy. Am J Ophthalmol 1976;82: 252-255.
21 Maloney WF, Robertson DM, Stuart M, Dubuff M: Hereditary vitelliform macular degeneration - Variable fundus findings within a single pedigree. Arch Ophthalmol 1977;95:979_ 983.

22 Merin S: Best's vitelliform macular dystrophy; in Merin S: Inherited Eye Diseases: Diagnosis and Clinical Management. New York, Marcel Dekker, 1991, pp 151-159.

23 Miller SA: Multifocal Best's vitelliform dystrophy. Arch Ophthalmol 1977;95:984-990.

24 Weingeist TA, Kobrin JL, Watzke RC: Histopathology of Bests's macular dystrophy. Arch Ophthalmol 1982;100:1108-1114.

25 O'Gorman S, Flaherty WA, Fishman GA, Berson EL: Histopathologic findings in Best's vitelliform macular dystrophy. Arch Ophthalmol 1988;106:1261-1268.

26 Arden GB, Barrada A, Kelsey JH: New clinical test of retinal function based upon the standing potential of the eye. Br J Ophtalmol 1962;46: 449-467.

27 Fishman GA, Trimble S, Rabb MF, Fishman M: Pseudovitteliform macular degeneration. Arch Ophthalmol 1977;95:73-76.

28 Oesterberg GA: Topography of the layer of rods and cones in the human retina. Acta Ophthalmol 1935; 13:1-102.

29 Rudolph G, Kalpadakis P, Bechmann M, La Rocca G, Hörmann C, Berninger T: Scanning laser ophthalmoscope-evoked multifocal-ERG (SLO-m-ERG) by using short m-sequences. Eur J Ophthalmol 2002;12:109-116.

30 Scholl HPN, Schuster AM, Vonthein R, Zrenner E: Mapping of retinal function in Best macular dystrophy using multifocal electroretinography. Vision Res 2002;42:1053-1061.

31 Seeliger M, Narfström K: Functional assessment of the regional distribution of disease in a cat model of hereditary retinal degeneration. Invest Ophthalmol Vis Sci 2000;41:19982005 . 